NOTES

\title{
Polypeptide Artificial Carrier Particles for Use in Passive Agglutination Immunoassay ${ }^{\dagger}$
}

\author{
Chuichi Hirayama*, Hirotaka Ihara, Masaaki Shibata, Takenori Hirai,* \\ Takeshi FujIYasu,* and Yoshihiro Yamauchi* \\ Department of Applied Chemistry, Faculty of Engineering, \\ Kumamoto University, Kumamoto 860, Japan \\ The Chemo-Sero-Therapeutic Research Institute, \\ Kikuchi Kyokushi, Kumamoto 861-15, Japan
}

(Received July 9, 1990)

\begin{abstract}
KEY WORDS Polyamino Acid / Glutamate / Microspheres / Coacervates / Immunoassay / Agglutination /
\end{abstract}

We previously reported that porous spherical particles could be produced from poly $(\gamma$-methyl L-glutamate) (PMLG) using a suspension and evaporation method. ${ }^{1}$ Since that time, these particles have been used for a wide range of applications, from GPC to affinity chromatography. ${ }^{1-8}$ Applications of the particles depend on their diameters, and are not restricted to liquid chromatography packings alone. For example, particles with $2-5 \mu \mathrm{m}$ diameters are widely used clinically as artificial carriers for immunoassay. Particles with $150-300 \mu \mathrm{m}$ diameters are used as carriers for cell culture. In the present report, we establish a method for producing spherical particles with diameters of $2-5 \mu \mathrm{m}$ from hydrophilic polyamino acid given below. Particles produced by this method were investigated for suitability as artificial carrier particles for immunoassay, and they were found to be excellent carriers, showing high sensitivity and a lack of nonspecific agglutination.

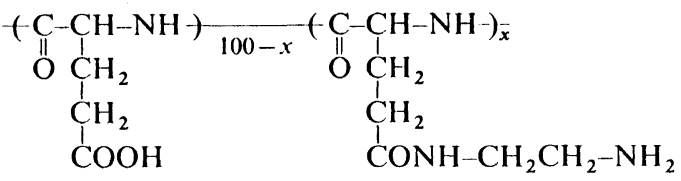

\footnotetext{
† Polypeptide Spheres VIII.
}

\section{EXPERIMENTAL}

\section{Preparation of Particles}

PMLG (with a degree of polymerization of 580, manufactured by Ajinomoto Co., Ltd.) prepared from the $N$-carboxyanhydride was reprecipitated from methanol, and $70 \mathrm{~g}$ of the PMLG powder obtained was suspended in $1000 \mathrm{ml}$ of a water-ethylene diamine mixture of a given ratio (Table I). After stirring over heat for $30-120 \mathrm{~min}$ at reflux temperature, a further $350 \mathrm{ml}$ of $2 \mathrm{wt} \%$ of $\mathrm{NaOH}$ aqueous solution was added, and stirring over heat was continued until the solution became clear. After filtering the undissolved portions, concentration in vacuo was performed to obtain a viscous material. This was dissolved in ethanol and partially aminated poly(L-glutamic acid) derivatives were obtained in the form of flaky substrances in ethyl ether. The amount of amino groups introduced was determined using titration and ${ }^{1} \mathrm{H}$ NMR spectra. ${ }^{1} \mathrm{H}$ NMR (in trifluoroacetic acid) $1.6-3.0 \mathrm{ppm}(\mathrm{m}), 3.2-$ $4.0 \mathrm{ppm}(\mathrm{m}), 4.1-5.1 \mathrm{ppm}(\mathrm{m})$.

$1.0 \mathrm{~g}$ of partially aminated poly(L-glutamic acid) was mixed with $20 \mathrm{ml}$ of $5 \mathrm{wt} \%$ gum arabic aqueous solution and $180 \mathrm{ml}$ of distilled 
water $1.5 \mathrm{ml}$ of $2 \mathrm{wt} \%$ Reactive Red 120 solution was then added. While stirring the compound solution at room temperature, $10 \mathrm{wt} \%$ acetic acid was gradually added, and coacervates were observed to form with lowering of the pH. $25 \mathrm{ml}$ of $25 \mathrm{wt} \%$ glutaraldehyde solution was added to the solution containing the coacervates, and the mixture was stirred at room temperature for $1 \mathrm{~h}$ at $40^{\circ} \mathrm{C}$ for $1 \mathrm{~h}$. After being collected by centrifugation, the stabilized particles were washed with deionized water.

\section{Immobilization of Antigen}

The particles stabilized with glutaraldehyde were suspended in $\mathrm{pH} 7.4$ phosphate buffer (PBS) containing $0.005 \%$ tannic acid to make the polymer content $1.25 \%$ stirred at $37^{\circ} \mathrm{C}$ for $30 \mathrm{~min}$, washed again with PBS, suspended in $40 \mu \mathrm{g} \mathrm{ml}^{-1}$ bovine serum albumin (BSA)/PBS solution, and stirred at $37^{\circ} \mathrm{C}$ for $30 \mathrm{~min}$. After being washed with PBS, the particles on which BSA was immobilized were stocked in PBS.

Hepatitis B core (HBc)-immobilized particles were made by a similar procedure, using HBc.

\section{Agglutination Test}

$25 \mu \mathrm{l}$ each of diluted anti-BSA antiserum (rabbit) was placed in each U-bottomed well of a plastic microtiter plate in a series of successive two times dilution starting from 100 times, $25 \mu \mathrm{l}$ each of $0.8 \mathrm{v} / \mathrm{v} \%$ dispersion of the BSA-immobilized particles was added into each well and the mixtures were allowed to stand for $1 \mathrm{~h}$ after being shaken for $3 \mathrm{~min}$. The agglutination patterns of particles were observed.

In the case of human serum, $0.1 \%$ BSA/PBS solution was used as a diluting solution.

\section{RESULTS AND DISCUSSION}

\section{Preparation of Microspheres}

Partially 3-aminoethylated poly(L-glutamic acid) was chosen as a hydrophilic polyamino
Table I. Preparation condition of microspheres from polyamino acid

\begin{tabular}{|c|c|c|c|c|}
\hline & \multicolumn{2}{|c|}{ Medium $^{\mathrm{a}} / \mathrm{ml}$} & \multirow[t]{2}{*}{ Conversion $^{\mathrm{b}}$} & \multirow{2}{*}{$\mathrm{pH}^{\mathrm{c}}$} \\
\hline & $\begin{array}{l}\text { Ethylene } \\
\text { diamine }\end{array}$ & Water & & \\
\hline 1 & 300 & 700 & 34 & 5.7 \\
\hline 2 & 500 & 500 & 51 & 8.3 \\
\hline 3 & 600 & 400 & 63 & 8.7 \\
\hline 4 & 700 & 300 & 74 & 8.9 \\
\hline 5 & 800 & 200 & 87 & 9.2 \\
\hline
\end{tabular}

a Amount per $70 \mathrm{~g}$ of polyamino acid.

b The ratio of amino groups to total residues after $50 \mathrm{~min}$.

c $\mathrm{pH}$ at which coacervates begin to form.

acid, and it was obtained by allowing PMLG to react with ethylene diamine. Typical preparation conditions are shown in Table I. The rate of introduction of amino groups can be controlled by the relative concentration of ethylene diamine with respect to PMLG and reaction time. In our research, the degree of conversion from methoxy groups into amino ones was controlled to lie in the range of 30 to $90 \%$, in consideration of the ease of preparation of polyamino acid coacervates.

The sphering of the polyamino acid was achieved by complex coacervation method. In general, counter ionic components are essential for the formation of complex coacervation in this method. In the present study, gum arabic was selected as a coacervation inducing agent, because addition of gum arabic to the aqueous medium promoted the formation of coacervates, although it was confirmed that the coacervates could be produced from aminated poly(L-glutamic acid) alone without additional counter ions. The aminated poly(L-glutamic acid) dissolved easily in aqueous solution containing gum arabic with a $\mathrm{pH}$ of 10 or higher. When the $\mathrm{pH}$ of the solution was lowered gradually adding acetic acid while stirring, polyamino acid coacervates were formed. It was found that the $\mathrm{pH}$ level at which coacervates began to form was dependent on 


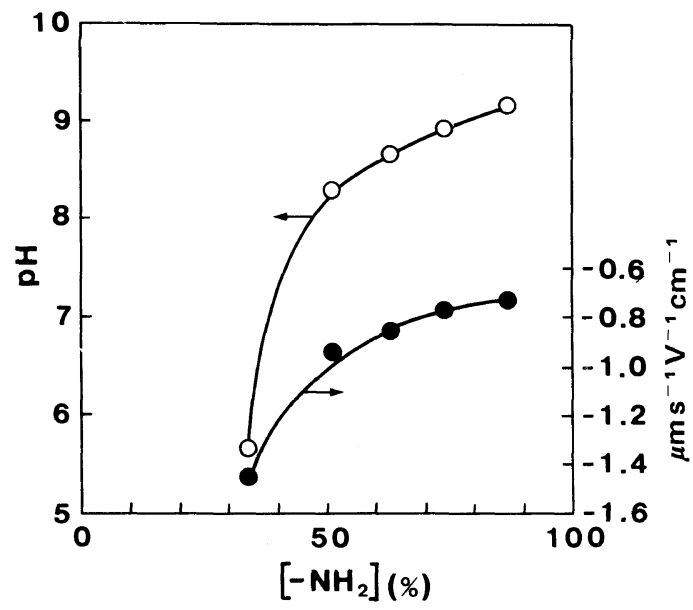

Figure 1. Relationships between the amount (\%) of amino groups introduced into poly(L-glutamic acid) and the $\mathrm{pH}$ value at which coacervates with the average diameter of $4 \mu \mathrm{m}$ are produced (-O-) and the electrophoretic mobility of particles (- - - ).

the introduction degree of the amino groups: the higher the introduction degree, the higher the $\mathrm{pH}$ level at which coacervates form. Figure 1 shows the relationship between the degree of conversion $(\%)$ into amino groups and the $\mathrm{pH}$ value at which coacervates with an average diameter of $4 \mu \mathrm{m}$ begin to be produced. It was determined by $\mathrm{pH}$ titration that the apparent $\mathrm{p} K_{\mathrm{a}} \mathrm{s}$ based on carboxylic and amino groups were about 4 and 8 , respectively. These results show that the formation of coacervates is related to the $\mathrm{p} K_{\mathrm{a}}$ of the amino groups of the polyamino acid.

The coacervates obtained were processed using glutaraldehyde, and the particles were stabilized and collected using centrifuging. The coacervates after treatment with $25 \mathrm{wt} \%$ glutaraldehyde for a few minutes no longer dissolved even at $\mathrm{pH} 1$ and 12, although non-treated coacervates dissolved with ease. In the case of sheep erythrocytes it takes about $2 \mathrm{~h}$ to stabilize with glutaraldehyde. This difference of stabilization time is attributable to a higher concentration of amino groups in coacervates from polyamino acids than in those of erythrocytes. It was observed that BSA could

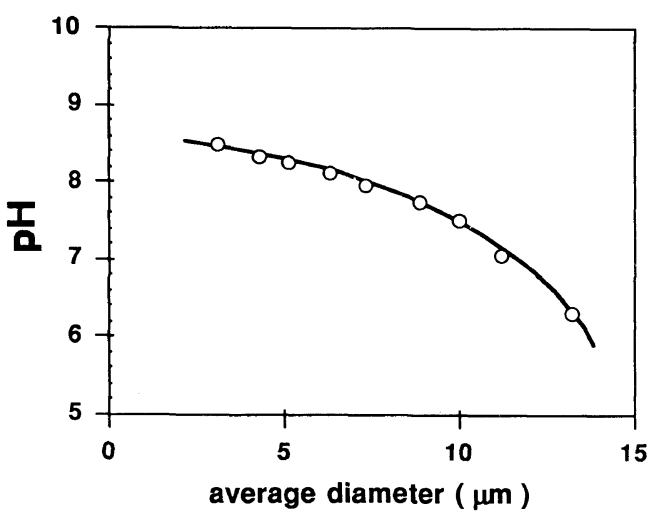

Figure 2. Relationship between the average diameter of polyamino acid particles and the $\mathrm{pH}$ in the coacervation of $51 \%$-aminated poly(L-glutamic acid).

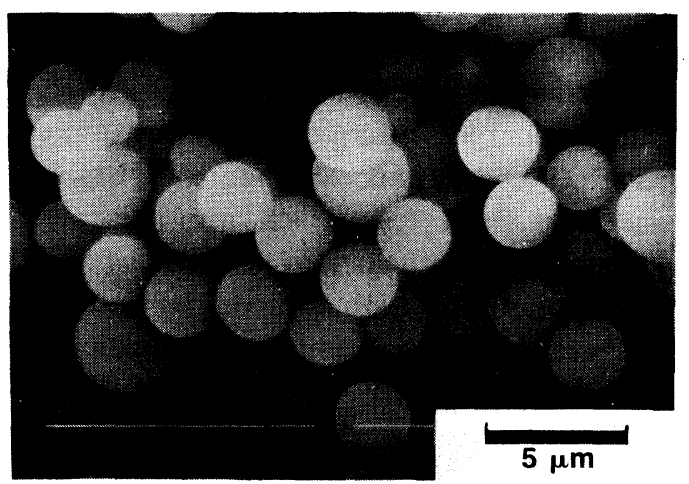

Figure 3. Typical electron micrograph of polyamino acid particles.

be easily immobilized under weak acidic condition onto the stabilized particles without further treatment. This indicates that active glutaraldehyde residues remaine on the particles.

The particle size produced was dependent on the $\mathrm{pH}$ level adjusted by acetic acid. The size distribution of particles which were treated with glutaraldehyde and washed with water and PBS was measured by light scattering method. ${ }^{9}$ For example, particles with average diameters of $4.2,8.9$ and $13.3 \mu \mathrm{m}$ were obtained at $\mathrm{pH} 8.3,7.8$, and 6.3 in the case of the $51 \%$-aminated poly(L-glutamic acid) as shown in Figure 2; this is related to the fact the lowering of $\mathrm{pH}$ by acetic acid induces 
polyamino acid lowered solubility. However, further lowering of $\mathrm{pH}$ to 4 or 5 caused aggregation of coacervates produced. As a result, it was found that $93-94 \mathrm{vol} \%$ or more of the particles have a size of $2-7 \mu \mathrm{m}$ due to proper selection of $\mathrm{pH}$ in the coacervation procedure. This size is considered appropriate for use as passive agglutination carrier particles for immunoassay. An electron micrograph of polyamino acid spherical particles obtained in this manner is shown in Figure 3.

In immunoassay by passive agglutination, the electrical charge condition of the particles plays an important role. In the case of polyamino acid particles, the particle charge is considered controllable by the relative ratio of the residual carboxyl groups of glutamic acid to the amino groups introduced. Further, the particle charge is inferred by the electrophoretic mobility of the particle (Figure 1). Stabilized sheep erythrocytes, which are presently in general use as carrier particles, have an electrophoretic mobility of $-1.16 \mu \mathrm{m} \mathrm{s}^{-1}$ $\mathrm{V}^{-1} \mathrm{~cm}^{-1}$. The polyamino acid particles obtained here showed a similar value of -0.73 to $-1.45 \mu \mathrm{m} \mathrm{s}^{-1} \mathrm{~V}^{-1} \mathrm{~cm}^{-1}$, depending on the degree of amination. ${ }^{10}$

\section{Immunoassay}

Non-specific agglutination of carrier particles is a disadvantageous characteristic for passive agglutination immunoassay. For example, it is known that polystyrene particles tend to adhere non-specifically to cells. ${ }^{11,12}$ Here, we investigated the agglutination of polyamino acid particles with non-sensitized stabilized sheep erythrocytes using normal human sera from 120 people. As shown in Figure 4, non-specific agglutination was observed in 57 cases $(47.5 \%$ of the sample) if the stabilized erythrocytes were mixed with the original serum. However, there was absolutely no agglutination with high dilution of serum in the erythrocytes. On the other hand, for polyamino acid particles, almost no nonspecific agglutination was observed under any

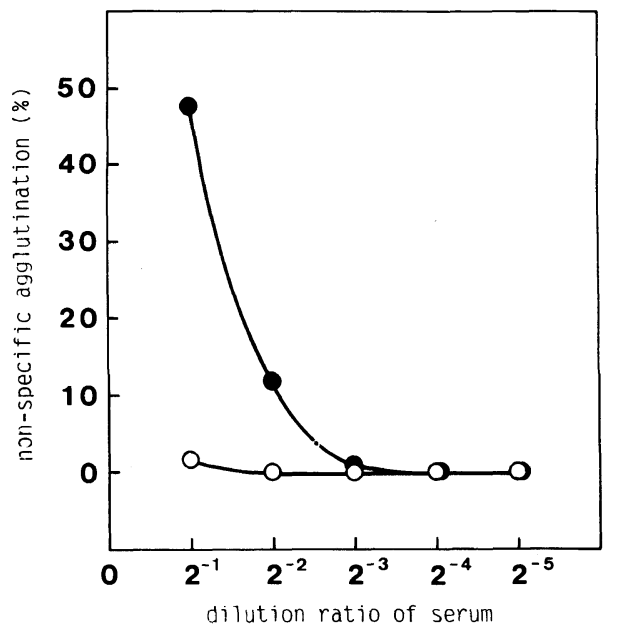

Figure 4. Relationship between the dilution ratio of human serum and the nonspecific agglutination (\%): - ${ }_{-}-$, polyamino acid particles; - — - stabilized sheep erythrocytes.

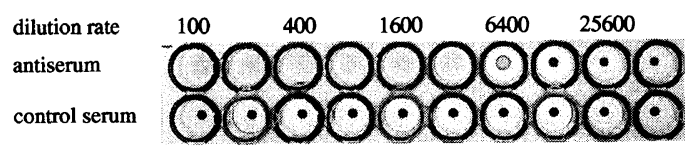

Figure 5. Agglutination patterns of BSA-immobilized polyamino acid particles reacted with anti-BSA antiserum on a microplate.

condition, clearly showing that the latter is more suitable for use as artificial carrier particles for immunoassay than the former.

In order to check the sensitivity in immunoassay, $\mathrm{HBc}$ and $\mathrm{BSA}$ were sensitized to the polyamino acid particles. A typical photograph of agglutination patterns is shown in Figure 5. It was determined that their sensitivity (potency) toward the respective antibodies was the same or higher than that of the stabilized erythrocytes. Detailed clinical results are forthcoming.

Also, it was found that the time required for assay was reduced approximately $2 / 3$ compared with that of stabilized sheep erythrocytes. This is due to the fact that the polyamino acid particles have a greater specific gravity as carriers. Their specific gravities were determined by cesium chloride isopycnic cen- 
trifugation method and the value was 1.30 in the erythrocytes and 1.44 in the polyamino acid particles, respectively.

In conclusion, polyamino acid particles show some advantages as passive agglutination carrier particles for immunoassay: (1) particle sizes and electric charge condition are controllable with ease, (2) it is easy to sensitize antigens (or antibodies), and (3) the particles has a lack of non-specific agglutination (it is an unavoidable property in carriers from natural products).

Acknowledgements. We are grateful to Messrs. Munehiro Saisho, Haruo Fujita and Takashi Imamura of the Chemo-Sero-Therapeutic Research Institute for their capable assistance, Dr. Eiich Abe of Government Industrial Research Institute, Kyushu for measurement of electrophoretic mobility, and Mr. Makoto Iwatsuki of Ajinomoto Co., Ltd. for providing material poly $(\gamma$-methyl L-glutamate).

\section{REFERENCES}

1. H. Ihara, T. Yoshinaga, and C. Hirayama, $J$. Chromatogr., 362, 197 (1986)

2. C. Hirayama, H. Ihara, S. Nagaoka, H. Furusawa, and S. Tsuruta, Polym. J., 22, 614 (1990).

3. C. Hirayama, H. Ihara, T. Yoshinaga, H. Hirayama, and Y. Motozato, J. Liq. Chromatogr., 9, 945 (1986).

4. S. Nagaoka, T. Urakawa, H. Ihara, and C. Hirayama, Polym. Prepr. Jpn., 39, 985 (1990).

5. S. Nagaoka, H. Ihara, and C. Hirayama, Polym. Prepr. Jpn., 38, 754 (1989).

6. H. Ihara, H. Furusawa, X. Li, and C. Hirayama, $J$. Appl. Polym. Sci., to be accepted.

7. C. Hirayama, H. Ihara, X. Li, K. Aihara, and S. Tsunoda, Kobunshi Ronbunsyu, 47, 119 (1990).

8. C. Hirayama, H. Ihara, and X. Li, J. Chromatogr., Biomed. Appl., 530, 148 (1990).

9. The diameters of coacervates were determined by use of the particle distribution analyzer CIS-1, Galai Co., Ltd.

10. After dispersing particles in $0.15 \mathrm{M}-\mathrm{PBS}(\mathrm{pH} 7.2)$, they were introduced into $1 \mathrm{~mm} \times 20 \mathrm{~mm}$ cylindrical electrophoresis cells and measured using a Penkem Co. Laser Zee system 3000 to produce a $25^{\circ} \mathrm{C}$, $1048 \mathrm{~V} \mathrm{~m}^{-1}$ gradient voltage.

11. A. Rembaum, Nature, 249, 81 (1974).

12. A. Rembaum, S. P. S. Yen, E. Cheong, S. Wallace, R. S. Molday, L. L. Gordon, and W. J. Dreyer, Macromolecules, 9, 328 (1976). 